\title{
Use of Cangrelor in Cervical and Intracranial Stenting for the Treatment of Acute Ischemic Stroke: A "Real Life" Single-Center Experience
}

\author{
(D) A. Cervo, (D) F. Ferrari, (D) G. Barchetti, (D) Quilici, (D). Piano, (DE. Boccardi, and (D) G. Pero
}

\begin{abstract}
BACKGROUND AND PURPOSE: In cases of large-vessel-occlusion strokes due to an underlying tandem internal carotid artery occlusion or intracranial atherosclerotic disease, concomitant stent placement may be needed. Immediate platelet inhibition is necessary, but to date, a standardized approach for antiplatelet inhibition in acute settings is still missing. Here we report our single-center experience about the safety and efficacy of periprocedural administration of cangrelor in patients with acute ischemic stroke due to intracranial or cervical artery occlusion undergoing stent placement.
\end{abstract}

MATERIALS AND METHODS: We retrospectively evaluated all cases of acute ischemic stroke that needed acute stent implantation and were treated with periprocedural administration of cangrelor between January 2019 and April 2020 at our institution. All patients who needed either extracranial or intracranial artery stent placement (in either the anterior or posterior circulation) were included.

RESULTS: We evaluated 38 patients in whom cangrelor was administered IV periprocedurally. Their mean age was 64 years (range, 26-85 years), with 25/38 male subjects and 13/38 female patients. In 26 patients (68.4\%), a tandem occlusion was present and was treated with carotid artery stent placement, while 12 patients (31.6\%) required an intracranial stent implantation. In 4 subjects (10.5\%), an intracerebral hemorrhage occurred after the procedure. All patients in the series were alive 1 week after the procedure.

CONCLUSIONS: Although larger, multicentric randomized studies are strongly warranted, our results support the hypothesis of a possible role of cangrelor as a valuable therapeutic option in the management of platelet inhibition in acute ischemic stroke procedures after intra- or extracranial stent placement.

ABBREVIATIONS: AIS = acute ischemic stroke; DAPT = dual-antiplatelet therapy; $\mathrm{GP}=$ glycoprotein; $\mathrm{ICH}=$ intracerebral hemorrhage; $\mathrm{PCI}=$ percutaneous coronary intervention; STEMI $=$ ST-segment elevation myocardial infarction

E dovascular treatment has become the standard care of acute ischemic stroke (AIS) due to large-vessel occlusion, which results most often from a cardioembolic event (mainly atrial fibrillation). ${ }^{1}$ Nevertheless, $15 \%-20 \%$ of large-vessel strokes have an underlying tandem internal carotid artery occlusion or intracranial atherosclerotic disease ${ }^{2}$, with the latter showing a higher incidence in the Asian population. ${ }^{3}$ Patients with AIS due to an underlying atherosclerotic extracranial or intracranial stenosis

Received May 6, 2020; accepted after revision July 7.

From the Department of Neuroradiology (A.C., F.F., L.Q., M.P., E.B., G.P.), Azienda Socio-Sanitaria Territoriale Grande Ospedale Metropolitano Niguarda, Milan, Italy Department of Biology and Biotecnology (F.F.), University of Pavia, Pavia, Italy; and Department of Neuroradiology (G.B.), Sapienza University, Rome, Italy.

Please address correspondence to Guglielmo Carlo Pero, MD, Piazza Ospedale Maggiore, 320162 Milan, Italy; e-mail: guglielmocarlo.pero@ospedaleniguarda.it

Indicates article with supplemental on-line table.

http://dx.doi.org/10.3174/ajnr.A6785 frequently need concomitant stent placement to maintain vessel patency.

Thrombosis is a known and serious complication following stent implantation. Indeed, both bare metal and drug-eluting stents can increase the thrombogenicity of atherosclerotic plaque, inducing platelet adhesion, activation, and thrombus formation directly on or near the stent. ${ }^{4}$ Thus, dual-antiplatelet therapy (DAPT) is long considered mandatory in patients undergoing endovascular stent placement, to reduce the risk of thromboembolic complications.

In emergency settings, when stent placement is required in patients without preadmission DAPT, the choice of the right antiplatelet treatment among all those available could be problematic. Thus, inhibitors of glycoprotein IIb/IIIa (GP IIb/IIIa) are known to be safe and efficient, being widely used in clinical practice. $^{5}$

Nevertheless, in patients with AIS, the risk of intracranial hemorrhage is considerably higher, especially if intravenous fibrinolytic 
therapy has been administered, either if oral P2Y12 receptor inhibitors or IV GP IIb/IIIa inhibitors are administered or not. ${ }^{6}$ In the unfortunate case of the occurrence of an intracerebral hemorrhage, the long half-life of these medications may represent a disadvantage in patient management. Nonetheless, this risk is generally outweighed by the potentially more severe consequences of vessel occlusion due to in-stent thrombosis.

Most of the antiplatelet strategies used in neurointerventions come from experience in interventional cardiology. Indeed, the use of DAPT in the emergency department has been extensively investigated in patients with ST-segment elevation myocardial infarction (STEMI) and without it. According to current guidelines, ${ }^{7}$ in patients with STEMI and in subjects referred to percutaneous coronary intervention (PCI), pretreatment with a $\mathrm{P} 2 \mathrm{Y} 12$ inhibitor is generally recommended in addition to aspirin (Bayer). In particular, ticagrelor administration is recommended on top of aspirin, regardless of the initial treatment strategy and also in patients already undergoing clopidogrel administration. According to the same guidelines, ${ }^{7}$ when patients undergoing a PCI are not pretreated with a P2Y12 inhibitor, cangrelor can be safely used in the catheterization laboratory and ticagrelor can be administered any time before, during, or at the end of cangrelor infusion.

Although characterized by these valuable features, cangrelor use is still off-label in the setting of neuroendovascular interventions.

Given the relative lack of evidence about cangrelor efficacy in the setting of acute neuroendovascular interventions, the aim of this study was to report a single-center experience about the effects of periprocedural IV administration of this drug in patients with AIS due to intracranial or cervical artery occlusion, who needed stent placement.

\section{MATERIALS AND METHODS \\ Patient Population}

This retrospective study was approved by Niguarda Cà Granda Hospital review board. Informed consent was secured from each patient, if possible, or from his or her next of kin.

At our institution, standard informed consent for emergency endovascular procedures includes consent to use antiplatelet drugs, if needed, even if off-label. Our institution approved the off-label use of cangrelor for neuroendovascular procedures.

Our single-center prospectively maintained data base was searched to evaluate all patients with AIS who underwent an endovascular procedure requiring acute stent implantation and who were treated with periprocedural administration of cangrelor. All patients who needed either extracranial or intracranial stent placement, both in the anterior and posterior circulations between January 2019 and April 2020, were included.

\section{Cangrelor Administration Protocol}

Our approach in the presence of AIS due to tandem occlusions is to treat the patient in a "retrograde fashion," performing intracranial thrombectomy first and stent placement afterward, with the patient under conscious sedation, if possible.

Imaging protocol for patient selection was achieved by the acquisition of a nonenhanced CT scan followed by multiphasic CTA and CT perfusion. Choice of an adequate thrombectomy technique (ie, a direct aspiration first-pass technique, stent-retriever, or a combination of both) was then made by each neuroradiologist according to the site of the occlusion. Balloon-guided catheters were used only in cases of intracranial ICA or cervical ICA occlusions with no concomitant intracranial occlusions.

The choice to implant a stent was made in the neurointerventional suite if no hemorrhagic complications occurred after thrombectomy and a good recanalization (TICI 2b-3) was achieved, according to the decision process shown in the Figure.

The only exclusion criterion for cangrelor administration was intracranial bleeding, ruled out by a conebeam CT performed in the angiosuite.

As soon as the operator deemed it necessary to implant a stent, in patients with no ongoing antiplatelet therapy and not receiving rtPA administration, a loading dose of $500 \mathrm{mg}$ of aspirin was administered intravenously at the beginning of the procedure. After stent placement, its patency was monitored for about 30 minutes, with the patient remaining in the angiosuite and angiograms were obtained every 10 minutes.

If no intrastent thrombosis occurred, cangrelor administration was avoided. On the other hand, in the case of intrastent thrombosis a $30-\mu \mathrm{g} / \mathrm{kg}$ bolus of cangrelor was administered, followed by a $4.0-\mu \mathrm{g} / \mathrm{kg} / \mathrm{min}$ IV infusion, until the transition to the

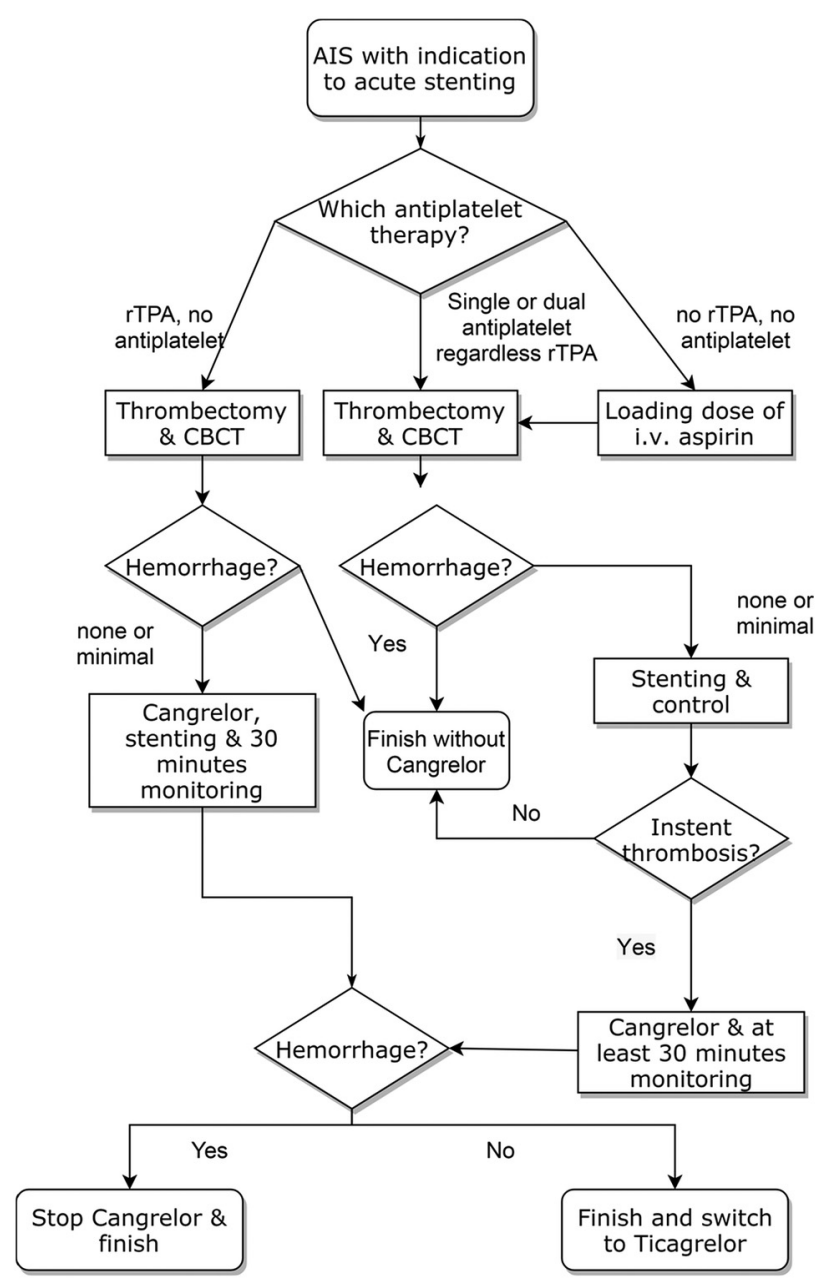

FIGURE. Stent placement and cangrelor administration decision process. CBCT indicates conebeam CT. 
oral P2Y12 inhibitor ticagrelor was deemed possible according to the treating physician. A loading dose of ticagrelor, $180 \mathrm{mg}$, was administered 30 minutes before cangrelor infusion was discontinued. No blood assay was performed to evaluate the effectiveness of antiaggregation, according to our institutional protocol. The postprocedural dual-antiplatelet therapy was performed with ticagrelor, $90 \mathrm{mg}$ twice a day, and aspirin, $100 \mathrm{mg}$, except for individualized modifications.

In patients who were initially treated with rtPA with no ongoing antiplatelet therapy, after ruling out hemorrhagic complications of the thrombectomy, cangrelor was administered immediately before stent placement, as described above. If during cangrelor infusion, patients showed a clinical deterioration due to hemorrhagic complications, cangrelor administration was stopped and ticagrelor administration was avoided.

\section{Follow-Up and Study Outcomes}

All patients received close clinical monitoring in a Stroke Unit or in a Neurologic Intensive Care Unit for at least 24 hours after the procedure. A nonenhanced brain CT scan was scheduled 24 hours after the procedure for all patients in the absence of signs of neurologic deterioration. Cervical carotid artery stent patency was assessed with a Doppler sonographic examination performed 24 hours after the procedure, while intracranial stent patency was assessed with an intracranial CTA. Finally, a brain CT perfusion scan was performed only in selected cases in which doubts about stent patency and/or signs of cerebral ischemia occurred.

The primary outcomes were the occurrence of in-stent thrombosis and the evidence of intracrerebral hemorrhage (ICH) both periprocedurally and during the first week after the treatment in in patients stented acutely.

\section{Statistical Analysis}

For this report, only descriptive statistics were performed. As for demographic data and baseline patient population characteristics, continuous variables were presented as mean or median, and categoric values, as absolute values and percentages. Statistical analyses were performed using R, Version 3.6.1 (http://www.r-project. org/). ${ }^{8}$

\section{RESULTS}

Between January 2019 and April 2020, four hundred thirteen endovascular procedures for AIS were performed in our center. Among these, 65 patients, $15.7 \%$, required stent implantation, with 38 subjects (59\%) administered IV cangrelor periprocedurally. A complete list of patient demographics, procedural characteristics, and posttreatment complications is presented in the On-line Table.

In this series, the average age was $64 \pm 13$ years (range, 26-85 years). Five patients received endovascular treatment under general anesthesia, while the remaining 33 were treated under conscious sedation. Furthermore, 10 patients $(26.3 \%)$ were treated with an aspiration technique and 19 subjects (50\%) underwent a combination of aspiration and stent retriever, while in 6 patients (15.8\%), both aspiration and a combined technique were used. Finally, in the 3 subjects without intracranial occlusions (7.9\%), cervical ICA stent placement was performed.
Six patients were treated during the coronavirus disease 2019 (COVID-19) pandemic, and 1 tested positive for this condition. In 11 patients (28.9\%), the onset time of the stroke was unknown, while for the remaining patients, the mean onset-to-groin time was $305 \pm 116.8$ minutes (range, 120-776 minutes). At the time of the endovascular treatment, 10 patients $(26.3 \%)$ were under single- or dual-antiplatelet therapy for concomitant pathologies, while only 1 subject (2.6\%) was under oral anticoagulation for atrial fibrillation. Of the 38 subjects, 16 patients (42.1\%) received IV thrombolysis, while 11 subjects (28.9\%) also received intraprocedural aspirin in anticipation of stent placement.

Twenty-six patients (68.4\%) presented with tandem occlusions and were treated with cervical or intrapetrous carotid artery stent placement. In this subgroup, 25 Carotid Wallstents (Boston Scientific) were implanted, and only 1 PRO-Kinetic Energy cobalt chromium coronary stent (Biotronik) was implanted for the treatment of a cervical vertebral artery occlusion. The remaining 12 patients (31.6.\%) required intracranial stent implantation, due to either atherosclerotic disease or dissection of intracranial arteries. In this group, we implanted 7 PRO-Kinetic Energy cobalt chromium coronary stents (Biotronik), 4 Solitaire AB stents (Medtronic), and 1 Neuroform Atlas Stent System (Stryker Neurovascular).

With the exception of a single case $(2.6 \%$, TICI $2 \mathrm{a})$, in all patients $(97.4 \%)$, a good or complete recanalization was achieved (TICI 2b/3).

At the short-term imaging follow-up, no intraprocedural stent occlusion occurred, and no postprocedural stent occlusion occurred within the first 24 hours except for 3 cases (7.9\%). Two of these patients, already under oral antiplatelet therapy with clopidogrel, were not switched to ticagrelor and experienced occlusion of the stent when cangrelor infusion was discontinued. One of them underwent a second endovascular procedure during which tirofiban administration and subsequent ticagrelor transition were performed, and the stent was proved patent at 1-week follow-up. The second patient, who was treated with stent placement for an MCA occlusion with an underlying stenosis, did not undergo a second endovascular procedure because he was transferred to a spoke hospital after the procedure; and by the time clinical deterioration was noticed, an ischemic lesion affected the entire MCA territory. The third patient, who underwent basilar artery stent placement for an atherosclerotic stenosis, experienced early postprocedural stent occlusion due to clot progression from the contralateral vertebral artery despite the correct administration of ticagrelor 30 minutes before stopping cangrelor infusion.

During the first week after the procedure, only 1 stent occlusion occurred (2.6\%). This patient was under dual-antiplatelet therapy with ticagrelor and aspirin and was switched to ticlopidine and aspirin 24 hours before neurologic symptoms relapsed. The patient underwent a second endovascular procedure, with subsequent cangrelor administration and ticagrelor transition, obtaining stent patency at 1-week follow-up.

In 2 patients (5.2\%), a vessel perforation with minimal subsequent subarachnoid hemorrhage occurred, but it was stable after the end of the procedure. In 4 subjects, a small amount of contrast media was found in the Sylvian fissure due to stent retriever traction, without clinical consequences. In such cases, there was no clinical deterioration and no need to stop cangrelor infusion. 


\begin{tabular}{lllll}
\hline \multicolumn{1}{c}{ Compound } & Clopidogrel & \multicolumn{1}{c}{ Prasugrel } & \multicolumn{1}{c}{ Ticagrelor } & Cangrelor \\
\hline Marketed name & Plavix & Effient (US) & Brilinta (US) & Kengreal (US) \\
& & Efient (EU) & Brilique or Possia (EU) & Kengrexal (EU) \\
Reversibility & Irreversible & Irreversible & Reversible & Reversible \\
Administration route & Oral & Oral & Oral & Intravenous \\
Frequency of administration & Once daily & Once daily & Twice daily & Bolus plus infusion \\
Onset of effect & $2-8$ hours & 30 min to 4 hours & 30 min to 4 hours & Immediate \\
Duration of effect & $5-7$ days & $7-10$ days & $3-5$ days & $30-60$ min \\
Halflife & 6 hours & 7 hours & 8 hours & $3-5$ min \\
Apparent volume of distribution & $6-72 \mathrm{~L}$ & $44-68 \mathrm{~L}$ & $88 \mathrm{~L}$ & $3.9 \mathrm{~L}$ \\
Plasma protein binding & $98 \%$ & $98 \%$ & $99.7 \%$ & $97 \%-98 \%$ \\
Prodrug & Yes & Yes & No & No \\
Influenced by genetic variation & Yes & No & No & No \\
\hline
\end{tabular}

${ }^{a}$ Modified from Aguilar-Salinas et al, ${ }^{11} 2019$, with permission from BMJ Publishing Group Ltd.

In 4 subjects (10.5\%), a basal ganglia reperfusion ICH occurred after the procedure. None of these patients received intravenous thrombolysis, with 1 subject (2.6\%) requiring surgical evacuation. The immediate withholding of cangrelor infusion allowed the performance of a safe operation. In the remaining 3 cases, the $\mathrm{ICH}$ induced a clinical deterioration, but no surgical evacuation was required, with a subsequent nonenhanced CT scan showing no progression of the ICH after cangrelor discontinuation. All 4 patients were not switched to ticagrelor oral antiplatelet therapy.

All hemorrhagic complications occurred in the ischemic territory. No new hemorrhagic complications, either systemic or intracranial, occurred in the first postprocedural week.

\section{DISCUSSION}

This is one of the few reports regarding the intraprocedural use of cangrelor in patients with AIS treated with stent implantation. Although they are being characterized by a descriptive evaluation of data, obtained without a control group, our results seem to suggest that cangrelor could be an effective drug in preventing stent thrombosis, not increasing the risk of hemorrhagic transformation, especially compared with other intraprocedural antiplatelet therapies. ${ }^{9,10}$ Furthermore, the very short half-life of cangrelor could allow an immediate interruption of the antiplatelet action if its discontinuation is needed in case of a hemorrhagic event.

To date, very few studies have been performed to evaluate cangrelor efficacy during emergency neuroendovascular procedures. ${ }^{11-14}$ Indeed, only 27 patients with AIS eligible for stent implantation and subsequently treated with cangrelor administration have been described in literature, ${ }^{11-13}$ and to the best of our knowledge, our study describes, to date, the largest series of patients with AIS undergoing acute stent placement and cangrelor administration.

Until the end of 2018, ReoPro (abciximab) has been the main antiplatelet drug used in our center after stent placement during AIS endovascular procedures. Shortly thereafter, this treatment option was withdrawn from the italian drug market, and there was a need for a new antiplatelet therapy. Although valuable alternatives to abciximab (namely, tirofiban or eptifibatibe, both GP IIb/IIIa inhibitors) were considered at the time, cangrelor gained particular attention due to its promising results in endovascular cardiology procedures.

Cangrelor is a reversible inhibitor of the P2Y12 receptor, being a nonthienopyridine adenosine triphosphate analog. The main pharmacokinetic features of this drug are summarized in the Table.
In particular, compared with other P2Y12 inhibitors, cangrelor has a rapid onset of action, a short half-life, and a predictable pharmacokinetic profile, and it is extensively metabolized by plasma dephosphorylation to inactive metabolites. ${ }^{15}$ It has been approved by the Food and Drug Administration for IV periprocedural use in patients undergoing PCI not treated with a P2Y12 platelet inhibitor or glycoprotein IIb/IIIa inhibitors. For this indication, cangrelor is administered as a $30-\mu \mathrm{g} / \mathrm{kg}$ bolus, followed by a continuous infusion of $4 \mu \mathrm{g} / \mathrm{kg} / \mathrm{min}$ for the PCI duration or at least for 2 hours. The A Clinical Trial Comparing Cangrelor to Clopidogrel Standard of Care Therapy in Subjects Who Require Percutaneous Coronary Intervention (CHAMPION-PHOENIX) trial showed that the occurrence of adverse events at 48 hours (including myocardial infarction, ischemia-driven revascularization, stent thrombosis, and, in some cases, death) is lower in patients treated with cangrelor compared with those receiving clopidogrel. ${ }^{16}$

Given the growing evidence supporting the use of this drug in patients with STEMI undergoing PCI, some investigators have started to test its efficacy and safety in neurointerventional procedures. $^{11-14}$

Our results are in accordance with those reported by AguilarSalinas et $\mathrm{al}^{11}$ describing, in a small case series, patients undergoing emergency stent placement for acute ischemic stroke or symptomatic intracranial aneurysms treated with periprocedural cangrelor at half of the standard dose for PCI. Similarly, another study ${ }^{14}$ also reported the efficacy of a full dose of this drug in the antiplatelet management of the acute stroke phase in either stent-assisted coiling or flow-diverter treatment of intracranial aneurysms. Furthermore, our results are also in accordance with those reported by 2 other studies ${ }^{12,13}$ describing 2 case series of patients with AIS treated with stent implantation and cangrelor infusion. Finally, and also in line with previous evidence, the very low rate of symptomatic intracranial hemorrhage we found in our cases is similar to those in other reported series using cangrelor after stent placement. ${ }^{12,13}$

Nevertheless, some degree of heterogeneity exists in the literature regarding the administered cangrelor dose. In this study, we have administered the standard cardiologic PCI dose, similar to what happened in most of the previous case series. ${ }^{12,13}$ Nevertheless, another study reported using a half-dose of cangrelor; although only 8 patients were enrolled in their series, the authors reported neither hemorrhagic nor thromboembolic complications. ${ }^{11}$ In our study, the full-dose decision was made because the vessels of patients with 
AIS are likely to have unstable atheromatic plaques, similar to the atheromatous coronary arteries that are PCI targets. On the other hand, ruptured aneurysms (predominantly dissecting if acute stent placement is required) entail an intrinsically higher risk of hemorrhagic complications compared with AIS. Although the main purpose of this study was to report our experience with cangrelor in the treatment of patients with AIS without giving directives about dosage and administration protocol, we think that future studies focusing on different doses of this drug are warranted to find the right balance between efficacy in avoiding thromboembolic complications and reduction of hemorrhagic complications and, in the future, also obtaining tailored drug doses in different patients.

As previously mentioned, cangrelor has a pharmacokinetic profile, especially compared with GP IIb/IIIa inhibitors, that is particularly fitted for its use in acute interventions, when fast, effective treatment and a short half-life are desired. Indeed, its infusion can be started during the procedure and immediately before stent placement without any premedication, a great advantage in neuroendovascular procedures in which a risk of intracranial vessel injury resulting in hemorrhagic complications is present. In fact, due to the fast effect of cangrelor, the operator can rapidly opt for stent placement and antithrombotic therapy up until the end of the intracranial maneuvers, after ruling out any intracranial hemorrhage.

Furthermore, in those few cases in which hemorrhagic complications occur during cangrelor infusion, its short half-life makes the case management easier, given that its antiplatelet activity subsides in less than an hour after the end of the infusion. ${ }^{16}$ This feature is of great importance, especially in cases in which a rapid hematoma evacuation is needed, eliminating the need for platelet transfusion. On the other hand, if hemorrhagic complications occur during either GP IIb/or P2Y12 inhibitor treatment, a platelet transfusion may be needed to correct platelet activity, with subsequent time loss and a higher risk of hemorrhage progression. ${ }^{17}$

In our series, only 1 patient needed emergency surgical evacuation because of a large basal ganglia hematoma ruled out immediately after the procedure, while cangrelor infusion was still ongoing and promptly discontinued for surgical purposes. A similar case has been recently described, ${ }^{13}$ with a patient who received cangrelor as bridging therapy and required an urgent operation 72 hours after the procedure due to an enlarging infarction. Although some differences between the 2 cases exist (eg, indications or time of surgery), in both cases, the management of the antiplatelet therapy with cangrelor proved to be a safe treatment, avoiding platelet transfusion.

Thus, cangrelor might be a valuable treatment option also as a bridging strategy (also at the low $0.75-\mu \mathrm{g} / \mathrm{kg} / \mathrm{min}$ dose), as recently reported. ${ }^{18}$ Indeed, in a case series recently reported by Godier et $\mathrm{al}^{19}$ on patients undergoing an unplanned operation after intracranial stent placement, the bridging protocol succeeded in achieving and maintaining platelet inhibition during DAPT discontinuation, with a return to baseline platelet function within 1 hour after cangrelor withdrawal.

Finally, cangrelor might be considered a first-line agent in patients with chronic kidney disease. ${ }^{20}$ Indeed, it is known that renal function does not alter the pharmacokinetic profile of the drug, ${ }^{21}$ even if clinical studies selectively performed on this population of patients have not been published yet. In this context, in several patients with AIS, there is usually a concomitant presence of renal dysfunction. Given that these patients usually receive high contrast media doses, both during the diagnostic workflow and the endovascular procedure, having a treatment option that does not affect the renal function is desirable.

Once cangrelor is infused or suspended, ticagrelor (loading dose, $180 \mathrm{mg}$ ) has been found to be the safest drug for maintaining antiplatelet activity; it binds competitively to the P2Y12 receptors, thus allowing a continuous and more predictable antiplatelet effect. ${ }^{22,23}$

Different studies have shown a possible benefit of ticagrelor when switching from cangrelor. ${ }^{22,23}$ In our series, 2 subjects were under clopidogrel treatment at the time of the procedure. Given the lack of studies on patients treated simultaneously with clopidogrel and cangrelor, we refrained from switching to ticagrelor in these 2 patients to avoid an increase in the risk of intracranial hemorrhage. Furthermore, previous studies postulated a competitive effect of cangrelor on clopidogrel antiplatelet activity, thus inducing a blockage of P2Y12, making it unavailable for the active metabolite of clopidogrel itself. ${ }^{23,24}$

These considerations are also indirectly confirmed and substantiated by some of our results. In particular, in 2 cases of early in-stent thrombosis we experienced, the relapse of neurologic symptoms was documented approximately 50 minutes after the switch from cangrelor to aspirin and clopidogrel, which incidentally appears to be the duration of cangrelor effect.

The 4 patients showing postprocedural ICH in our series had poor blood pressure control following the endovascular treatment. Although there is no clear correlation between poor blood pressure control and hemorrhagic complications, existing data suggest that uncontrolled blood pressure might be a relevant risk factor for ICH development after carotid artery stenosis correction. Strict monitoring of blood pressure (with systolic blood pressure values of $<140 \mathrm{~mm} \mathrm{Hg}$ ) is therefore recommended in the angiosuite as well as during the first postoperative week to avoid reperfusion hemorrhage. ${ }^{25}$

On the other hand, if a small and self-limiting subarachnoid hemorrhage suffusion occurs, cangrelor could be safely continued under clinical and imaging monitoring because this complication is often self-limiting.

This study has several limitations, mostly related to its retrospective, single-center nature, with a relatively small number of patients enrolled, with short follow-up, and without comparison with a control group. In addition, we did not perform any test to evaluate the platelet response to P2Y12 inhibition. Finally, in our group, there was a clear under-representation of patients already treated with oral anticoagulants, which usually represent a significant proportion of patients undergoing emergent neuroendovascular procedures, therefore limiting the generalizability of our results.

\section{CONCLUSIONS}

Although it is characterized by these limitations, we here report a relatively large case series about periprocedural administration of cangrelor in patients with AIS following the occlusion of an intracerebral or cervical artery and requiring extracranial or intracranial stent placement. Even though larger and randomized studies are recommended, our results are in line with other preliminary observations suggesting that cangrelor might be a valuable therapeutic option in the management of emergency 
neuroendovascular interventions, given its efficacy in rapid platelet function inhibition and its fast resolution in cases of hemorrhagic complications.

\section{ACKNOWLEDGMENTS}

The authors thank Sirio Cocozza, MD, for his valuable assistance in the revision of the scientific English of this article.

DISCLOSURES: Federica Ferrari-RELATED: Other: scholarship from the Enrico ed Enrica Sovena Foundation, Rome, Italy.

\section{REFERENCES}

1. Powers WJ, Rabinstein AA, Ackerson T, et al; American Heart Association Stroke Council. Guidelines for the Early Management of Patients with Acute Ischemic Stroke: A Guideline for Healthcare Professionals from the American Heart Association/American Stroke Association. Stroke 2018;49:e46-110 CrossRef Medline

2. Grigoryan M, Haussen DC, Hassan AE, et al. Endovascular treatment of acute ischemic stroke due to tandem occlusions: large multicenter series and systematic review. Cerebrovasc Dis 2016;41:306-12 CrossRef Medline

3. Holmstedt CA, Turan TN, Chimowitz MI. Atherosclerotic intracranial arterial stenosis: risk factors, diagnosis, and treatment. Lancet Neurol 2013;12:1106-14 CrossRef Medline

4. Windecker S, Meier B. Late coronary stent thrombosis. Circulation 2007;116:1952-65 CrossRef Medline

5. Farrokh S, Owusu K, Lara LR, et al. Neuro-interventional use of oral antiplatelets: a survey of neuro-endovascular centers in the United States and review of the literature. J Pharm Pract 2019 Jul 21. [Epub ahead of print] CrossRef Medline

6. Zhu F, Anadani M, Labreuche J, et al; TITAN Investigators. Impact of antiplatelet therapy during endovascular therapy for tandem occlusions: a collaborative pooled analysis. Stroke 2020;51:1522-29 CrossRef Medline

7. Valgimigli M, Bueno H, Byrne RA, et al; ESC National Cardiac Societies. 2017 ESC focused update on dual antiplatelet therapy in coronary artery disease developed in collaboration with EACTS: the Task Force for dual antiplatelet therapy in coronary artery disease of the European Society of Cardiology (ESC) and of the European Association for Cardio-Thoracic Surgery (EACTS). Eur Heart J 2018;39:213-60 CrossRef Medline

8. R Core Team. R: A Language and Environment for Statistical Computing. R Foundation for Statistical Computing. 2019. https:// www.R-project.org/. Accessed May 5, 2020

9. Al-Mufti F, Amuluru K, Manning NW, et al. Emergent carotid stenting and intra-arterial abciximab in acute ischemic stroke due to tandem occlusion. Br J Neurosurg 2017;31:573-79 CrossRef Medline

10. Pan X, Zheng D, Zheng Y, et al. Safety and efficacy of tirofiban combined with endovascular treatment in acute ischaemic stroke. Eur J Neurol 2019;26:1105-10 CrossRef Medline

11. Aguilar-Salinas P, Agnoletto GJ, Brasiliense LBC, et al. Safety and efficacy of cangrelor in acute stenting for the treatment of cerebrovascular pathology: preliminary experience in a singlecenter pilot study. J Neurointerv Surg 2019;11:347-51 CrossRef

12. Linfante I, Ravipati $\mathrm{K}$, Starosciak $\mathrm{AK}$, et al. Intravenous cangrelor and oral ticagrelor as an alternative to clopidogrel in acute intervention. J Neurointerv Surg 2000 May 15. [Epub ahead of print] CrossRef Medline

13. Elhorany M, Lenck S, Degos V, et al. Cangrelor and stenting in acute ischemic stroke: monocentric case series. Clin Neuroradiol 2020 May 7. [Epub ahead of print] CrossRef Medline

14. Abdennour L, Sourour N, Drir M, et al. Preliminary experience with cangrelor for endovascular treatment of challenging intracranial aneurysms. Clin Neuroradiol 2019 Jul 15. [Epub ahead of print] CrossRef Medline

15. Storey RF, Sanderson HM, White AE, et al. The central role of the $\mathbf{P}$ (2T) receptor in amplification of human platelet activation, aggregation, secretion and procoagulant activity. Br J Haematol 2000;110:92534 CrossRef Medline

16. Bhatt DL, Stone GW, Mahaffey KW, et al; CHAMPION PHOENIX Investigators. Effect of platelet inhibition with cangrelor during PCI on ischemic events. N Engl J Med 2013;368:1303-13 CrossRef Medline

17. Lipinski MJ, Lee RC, Gaglia MA, et al. Comparison of heparin, bivalirudin, and different glycoprotein IIb/IIIa inhibitor regimens for anticoagulation during percutaneous coronary intervention: a network meta-analysis. Cardiovasc Revasc Med 2016;17:535-45 CrossRef Medline

18. Bhattad VB, Gaddam S, Lassiter MA, et al. Intravenous cangrelor as a peri-procedural bridge with applied uses in ischemic events. Ann Transl Med 2019;7:408 CrossRef Medline

19. Godier A, Mesnil M, De Mesmay M, et al. Bridging antiplatelet therapy with cangrelor in patients with recent intracranial stenting undergoing invasive procedures: a prospective case series. $\mathrm{Br} J$ Anaesth 2019;123:e2-e5 CrossRef Medline

20. Van Tuyl JS, Newsome AS, Hollis IB. Perioperative bridging with glycoprotein IIb/IIIa inhibitors versus cangrelor: balancing efficacy and safety. Ann Pharmacother 2019;53:726-37 CrossRef Medline

21. Neumann FJ, Hochholzer W, Pogatsa-Murray G, et al. Antiplatelet effects of abciximab, tirofiban and eptifibatide in patients undergoing coronary stenting. J Am Coll Cardiol 2001;37:1323-28 CrossRef Medline

22. Schneider DJ, Agarwal Z, Seecheran N, et al. Pharmacodynamic effects during the transition between cangrelor and ticagrelor. JACC Cardiovasc Interv 2014;7:435- 42 CrossRef Medline

23. Steinhubl SR, Oh JJ, Oestreich JH, et al. Transitioning patients from cangrelor to clopidogrel: pharmacodynamic evidence of a competitive effect. Thromb Res 2008;121:527-34 CrossRef Medline

24. Dovlatova NL, Jakubowski JA, Sugidachi A, et al. The reversible P2Y12 antagonist cangrelor influences the ability of the active metabolites of clopidogrel and prasugrel to produce irreversible inhibition of platelet function. J Thromb Haemost 2008;6:1153-59 CrossRef Medline

25. Farooq MU, Goshgarian C, Min J, et al. Pathophysiology and management of reperfusion injury and hyperperfusion syndrome after carotid endarterectomy and carotid artery stenting. Exp Transl Stroke Med 2016;8:7 CrossRef Medline 